\title{
The Applications of a Newton-Type Method for Constrained Nonsmooth Equations
}

\author{
D. Y. Pang, S.Q. Du, J.J. Ju \\ College of Mathematics, Qingdao University \\ Qingdao, China
}

\begin{abstract}
In this paper, we give some applications of a Newtontype method for constrained nonsmooth equations. The method is proposed by F.Facchinei and etc, which use linear programming model for the solution of constrained nonsmooth equations. The applications of the method including in solving constrained maximum equations and generalized complementarity problems.
\end{abstract}

Keywords-constrained nonsmooth equation; newton-type method; linear programming

\section{INTRODUCTION}

We consider the constrained nonsmooth equations

$$
F(x)=0, x \in \Omega,
$$

where $\Omega \subseteq R^{n}$ is a nonempty and closed set and $F: R^{n} \rightarrow R^{m}$ is locally Lipschitz function. The solution set of (1) is denoted by $X$ and assumed to be nonempty, that is

$$
X=\{x \in \Omega \mid F(x)=0\} \neq \Phi .
$$

Numerical analysis methods for solving (1) have been studied by many researchers, such as [1-7]. In [1], they consider a new Newton-type methods, when given a point $x^{k} \in \Omega$, under a mild assumption, $x^{k+1}$ is a feasible solution of the following linear programming problem

$$
\begin{gathered}
\min _{x, \gamma} \gamma \\
\left\|F\left(x^{k}\right)+G\left(x^{k}\right)\left(x-x^{k}\right)\right\| \leq \gamma\left\|F\left(x^{k}\right)\right\|, \\
\left\|x-x^{k}\right\| \leq \gamma\left\|F\left(x^{k}\right)\right\|, \\
x \in \Omega, \gamma \geq 0,
\end{gathered}
$$

where $G\left(x^{k}\right)$ is an element of a subdifferential.

To describe the scheme of the method, for given $s \in \Omega$ and $\gamma \geq 0$, define the set

$$
\mathrm{P}(s, \gamma):=\left\{x \in \Omega \mid\|F(s)+G(s)(x-s)\| \leq \gamma\|F(s)\|^{2},\|x-s\| \leq \gamma\|F(s)\|\right\} .
$$

where $\|\cdot\|$ is an arbitrary but fixed norm in $R^{n}$ or $R^{m}$. Now, as in [1], we give the scheme of the Newton-type method for (1).

\section{Method 1.}

Step 0: Choose $x^{0} \in \Omega$ and $\Gamma>0$. Let $k:=0$.
Step 1: If $x^{k} \in X$,then stop.

Step 2: Solve the $x^{k+1} \in \mathrm{P}\left(x^{k}, \Gamma\right)$.

Step 3: Set $k:=k+1$. go to Step 1 .

In the following of the paper, we give the applications of the Method 1 in section 2. In section 3, some discussions of the method are also given.

\section{APPLICATIONS OF THE METHOD}

A. Application in Solving Constrained Maximum Equations

In this section, we consider the constrained maximum equations

$$
\max _{j \in J_{1}} f_{1 j}(x)=0
$$

$\vdots$

$\max _{j \in J_{n}} f_{n j}(x)=0, x \in \Omega$,

where $\Omega \subseteq R^{n}$ is a nonempty and closed set, $f_{i j}: R^{n} \rightarrow R$ are contiously differentiable functions, $j \in J_{i}, i=1, \ldots, n$, $J_{i}$ are finite index sets. Obviously, (3) is a system of nonsmooth equations. For simplicity, we denote

$$
\begin{gathered}
f_{i}(x)=\max _{j \in J_{i}} f_{i j}(x), \quad x \in \Omega \quad i=1, \ldots, n \\
F(x)=\left(f_{1}(x), \ldots, f_{n}(x)\right)^{T}, \quad x \in \Omega \\
J_{i}(x)=\left\{j \in J_{i} \mid f_{i j}(x)=f_{i}(x)\right\}, x \in \Omega, \quad i=1, \ldots, n .
\end{gathered}
$$

Thus, (3) can be briefly written as

$$
F(x)=0, \quad x \in \Omega .
$$

Now, we introduce a new kind of the subdifferential for $F$ as in [2]. We denote it by $\partial_{*} F(x)$, which is given as

$$
\partial_{*} F(x)=\left\{\left(\nabla f_{1 j_{1}}(x), \ldots, \nabla f_{n j_{n}}(x)\right)^{T} \mid j_{1} \in J_{1}(x), \ldots, j_{n} \in J_{n}(x)\right\} .
$$

Based on Method 1, we give a new method for solving (3). Method 2.

Step 0: Choose $x^{0} \in \Omega$ and $\Gamma>0$. Let $k:=0$.

Step 1: If $x^{k} \in X$, then stop. 
Step 2: Solve the following linear programming problem to get $x^{k+1}$

$$
\begin{gathered}
\min _{x, \gamma} \gamma \\
\left\|F\left(x^{k}\right)+G\left(x^{k}\right)\left(x-x^{k}\right)\right\| \leq \gamma\left\|F\left(x^{k}\right)\right\|, \\
\left\|x-x^{k}\right\| \leq \gamma\left\|F\left(x^{k}\right)\right\|, \\
x \in \Omega, \gamma \geq 0,
\end{gathered}
$$

where $G\left(x^{k}\right) \in \partial_{*} F\left(x^{k}\right)$, that is $x^{k+1} \in \mathrm{P}\left(x^{k}, \Gamma\right)$.

Step 3: Set $k:=k+1$, go to Step 1 .

Let $x^{*} \in X, \beta\left(x^{*}, \delta\right):=\left\{x \in R^{n}\left\|x-x^{*}\right\| \leq \delta\right\}$, where $\delta>0$ is arbitrary constant, $\operatorname{dist}[s, X]:=\inf _{x \in X}\|s-x\|$ denote the distance of $S$ to $X$.

Proposition 1. If $F$ is locally Lipschitz continuous around $x^{*}$, that is, there exits $L>0$ such that

$$
\|F(s)\| \leq L . d i s t[s, X]
$$

holds for all $s \in \beta\left(x^{*}, \delta\right) \cap \Omega$.

Because Method 2 is not well-defined if $\mathrm{P}\left(x^{k}, \Gamma\right)$ is empty, to avoid this and prove the local quadratic convergence, according to [1], we give the following similar assumptions.

Assumption 1. There exists $l>0$ such that

$$
\operatorname{dist}[s, X] \leq l\|F(s)\|
$$

holds for all $s \in \beta\left(x^{*}, \delta\right) \cap \Omega$.

Assumption 2. There exists $\stackrel{-}{\Gamma} \geq 1$ such that

$$
\gamma(s) \leq \bar{\Gamma}
$$

holds for all $s \in \beta\left(x^{*}, \delta\right) \cap \Omega$, where $x^{k}$ was substituted by $s$ in $\gamma(s), \gamma(s)$ is the optimal value of (9).

Assumtion 3. There exists $\hat{\alpha}>0$ such that

$$
w \in \psi(s, \alpha):=\left\{w \in \Omega\|\| w-s\|\leq \alpha,\| F(s)+G(s)(w-s) \| \leq \alpha^{2}\right\}
$$

implies

$$
\|F(w)\| \leq \hat{\alpha} \alpha^{2}
$$

for all $s \in\left(\beta\left(x^{*}, \delta\right) \cap \Omega\right) \backslash X$ and all $\alpha \in[0, \delta]$.

To get the convergence of the Method 2, we need to give the following two lemma firstly.

Lemma 1. Let Assumption 2 be satisfied and define the set

$$
\mathrm{P}(s, \Gamma):=\left\{x \in \Omega|\|F(s)+G(s)(x-s)\| \leq \Gamma|\|F(s)\|^{2},\|x-s\| \leq \Gamma\|F(s)\|\right\}
$$

Then, for any $s \in \beta\left(x^{*}, \delta\right) \cap \Omega, \mathrm{P}(s, \Gamma)$ is nonempty. And we know that

$$
\begin{gathered}
\|F(s)+G(s)(x-s)\| \leq \Gamma L^{2} \operatorname{dist}[s, X]^{2}, \\
\|X-s\| \leq \Gamma L \operatorname{dist}[s, X]
\end{gathered}
$$

holds for all $x \in \mathrm{P}(s, \Gamma)$.

Lemma 2. Let Assumptions 1-3 be satisfied. Then, there are $\varepsilon>0$ and $c>0$ such that, for any $s \in \beta\left(x^{*}, \delta\right) \cap \Omega$,

$$
\operatorname{dist}[x, X] \leq \operatorname{cdist}[s, X]^{2} \leq \frac{1}{2} \operatorname{dist}[s, X]
$$

holds for all $x \in \mathrm{P}(s, \Gamma)$.

The proof of lemma 1 and lemma 2, we can see in [3].

Based on the above lemmas, we can get the convergence theorem of Method 2.

Theorem 1. Let Assumptions 1-3 be satisfied for some $\delta>0$ and let $\Gamma \geq \bar{\Gamma}$ be given. Then, if $x^{0} \in \beta\left(x^{*}, r_{\Gamma}\right) \cap \Omega$ and there is $r_{\Gamma} \in(0, \delta]$, we have

(i) Method 2 is well-defined (that is, $\mathrm{P}\left(x^{k}, \Gamma\right) \neq \Phi$ in step 2 for all $k$ ).

(ii) If $x^{0}, \ldots, x^{k}$ are generated by Method 2, then $x^{k+1} \in \beta\left(x^{*}, \delta\right) \cap \Omega$.

(iii) Any $\left\{x^{k}\right\}$ generated by Method 2 either stops with a solution or converges locally quadratically to some solution of (7).

Proof We can get (i) from Lemma 1. Now we prove (ii). By Method 1 is well-defined for any $x^{0} \in \Omega$, with $\varepsilon$ according to

$$
0<\varepsilon \leq \frac{1}{2} \min \left\{\delta, \delta \Gamma^{-1} L^{-1}, \alpha^{-1} l^{-1} \hat{\Gamma}^{-2} L^{-2}\right\} \cdot
$$

We can choose $r$ such that

$$
0<r<\frac{\varepsilon}{1+2 \Gamma L} .
$$

We first prove by induction that

$$
x^{k} \in \beta\left(x^{*}, \varepsilon\right) \cap \Omega
$$

and

$$
x^{k+1} \in \mathrm{P}\left(x^{k}, \Gamma\right)
$$

are holds for all $k \in N$.

For $k=0$, due to $x^{0} \in \beta\left(x^{*}, r\right) \cap \Omega, r<\varepsilon$, so $x^{0} \in \beta\left(x^{*}, \varepsilon\right) \cap \Omega$.Moreover, since $r<\varepsilon<\delta$ due to $0<\varepsilon \leq \frac{1}{2} \min \left\{\delta, \delta \Gamma^{-1} L^{-1}, \alpha^{-1} l^{-1} \hat{\Gamma}^{-2} L^{-2}\right\} \quad, \quad$ Assumption 2 implies $x^{1} \in \mathrm{P}\left(x^{0}, \Gamma\right)$. Suppose (10) and (11) hold 
for $k=0, \ldots, v$. To prove $x^{v+1} \in \beta\left(x^{*}, \varepsilon\right) \cap \Omega$, we first note that

$$
\left\|x^{v+1}-x^{*}\right\| \leq\left\|x^{v}-x^{*}\right\|+\left\|x^{v+1}-x^{v}\right\| \leq\left\|x^{0}-x^{*}\right\|+\sum_{j=0}^{v}\left\|x^{j+1}-x^{j}\right\| \cdot
$$

From (10) and (11) for $k=0, \ldots, v$ and Lemma 1, we have

$$
\left\|x^{j+1}-x^{j}\right\| \leq \Gamma \operatorname{Ldist}\left[x^{j}, X\right]
$$

for $j=0, \ldots, v$. By (10), (11) and Lemma 2, we get

$$
\operatorname{dist}\left[x^{j}, X\right] \leq \frac{1}{2} \operatorname{dist}\left[X^{j-1}, X\right] \leq \ldots \leq\left(\frac{1}{2}\right)^{j} \operatorname{dist}\left[x^{0}, X\right]
$$

for $j=0, \ldots, v$. Therefore, from (12) and (13), we obtain

$$
\left\|x^{v+1}-x^{*}\right\| \leq r+\Gamma L \operatorname{dist}\left[x^{0}, X\right] \sum_{j=0}^{v}\left(\frac{1}{2}\right)^{j} \leq r+\Gamma L . r .2 \leq(1+2 \Gamma L) r \leq \varepsilon .
$$

We have $x^{v+1} \in \beta\left(x^{*}, \varepsilon\right) \cap \Omega$. This and Assumption 2 imply $x^{v+2} \in \mathrm{P}\left(x^{v+1}, \Gamma\right)$. Hence, (10) and (11) hold for $k=v+1$ and for $k \in N$. By (10), (11) and Lemma 2, we get

$$
\operatorname{dist}\left[x^{k+1}, X\right] \leq \operatorname{c.dist}\left[x^{k}, X\right]^{2} \leq \frac{1}{2} \operatorname{dist}\left[x^{k}, X\right]
$$

for $k \in N$. Then, we get

$$
\lim _{k \rightarrow \infty} \operatorname{dist}\left[x^{k}, X\right]=0
$$

for $j, k \in N$ and $k>j$. From Lemma 1, (11) and Lemma 2, we have

$$
\left\|x^{k}-x^{j}\right\| \leq \sum_{i=j}^{k-1}\left\|x^{i+1}-x^{i}\right\| \leq \Gamma \operatorname{Ldist}\left[x^{j}, X\right] \sum_{i=j}^{k-1}\left(\frac{1}{2}\right)^{i} \leq 2 \Gamma L \operatorname{dist}\left[x^{j}, X\right]^{\cdot}
$$

Due to (16), we know that $\left\{z^{k}\right\}$ is a Cauchy sequence (by the closeness) converges to some $\hat{x} \in X$. Finally, we prove the convergence rate. The use of (17) for $k+1$ instead of $j$ and $k+j$ instead of $k$ together with (15) leads to

$$
\left\|x^{k+j}-x^{k+1}\right\| \leq 2 \Gamma \operatorname{Ldist}\left[x^{k+1}, X\right] \leq 2 c \Gamma L \operatorname{dist}\left[x^{k}, X\right]^{2}
$$

for any $k, j \in N, j>1$. The limit for $j \rightarrow \infty$, we obtain

$$
\left\|\hat{x}-x^{k+1}\right\| \leq 2 c \Gamma L \operatorname{dist}\left[x^{k}, X\right]^{2} \leq 2 c \Gamma L\left\|\hat{x}-x^{k}\right\|^{2} .
$$

We finished the proof.

\section{B. Application In Solving Generalized Complementarity Problem}

In this section, we consider the constrained generalized complementarity problem, which is to find $x \in \Omega$, such that

$$
F(x) \geq 0 \quad G(x) \geq 0 \quad F(x)^{T} G(x)=0, \quad x \in \Omega,
$$

where $\quad F=\left(F_{1}, F_{2}, \ldots, F_{n}\right)^{T}, \quad G=\left(G_{1}, G_{2}, \ldots, G_{n}\right)^{T}$, $F_{i}: R^{n} \rightarrow R(i=1, \ldots, n) \quad$ and $\quad G_{i}: R^{n} \rightarrow R,(i=1, \ldots, n) \quad$ are continuously differentiable functions, $\Omega \subseteq R^{n}$ is a nonempty and closed set.

One of the most popular approaches for solving (19) is to reformulate it as a system of nonlinear equations. For any nonlinear complementarity problem (NCP) function $\varphi$, (19) is equivalent to the following equation

$$
H(x)=\left[\varphi\left(F_{1}(x), G_{1}(x)\right), \ldots, \varphi\left(F_{n}(x), G_{n}(x)\right)\right]^{T}=0 .
$$

Here, we list some popular used nonlinear complementarity problem (NCP) functions as in [4].

$$
\begin{gathered}
\varphi_{1}(a, b)=\min \{a, b\}, \\
\varphi_{2}(a, b)=\sqrt{a^{2}+b^{2}}-(a+b), \\
\varphi_{3}(a, b)=(a-b)^{+}-a, \\
\varphi_{4}(a, b)=\|(a, b)\|_{p}-(a+b) \quad(p>1), \\
\varphi_{5}(a, b)=\sqrt[p]{\theta\left(|a|^{p}+|b|^{p}\right)+(1-\theta)|a-b|^{p}}-(a+b) \quad(\theta \in[0,1], p>1), \\
\varphi_{6}(a, b)=\sqrt{\left[\sqrt{a^{2}+b^{2}}-(a+b)\right]^{2}+\alpha\left[(a b)_{+}\right]^{2}} \quad(\alpha>0) .
\end{gathered}
$$

Obviously, all the $\varphi_{i}(a, b)(i=1, \ldots, 6)$ is strongly semismooth functions. If we choose $\varphi_{6}$ in (20), we have

$$
H(x)=\left[H_{1}(x), \ldots, H_{i}(x), \ldots, H_{n}(x)\right]^{T}=0,
$$

where

$$
\begin{gathered}
H_{i}(x)=\varphi_{6}\left(F_{i}(x), G_{i}(x)\right) \\
=\sqrt{\left[\sqrt{F_{i}^{2}(x)+G_{i}^{2}(x)}-\left(F_{i}(x)+G_{i}(x)\right)\right]^{2}+\alpha\left[\left(F_{i}(x) G_{i}(x)\right)_{+}\right]^{2}} \\
(i=1, \ldots, n)(\alpha>0) .
\end{gathered}
$$

Let $\partial H(x)$ denote Clarke's generalized Jacobian of $H$ at $x \in \Omega . \partial H(x)$ is a matrix which element is composed by every $\partial H_{i}(x)(i=1, \ldots, n)$. According to [4], we know that for any $i \in\{1,2, . ., n\}$ and $v \in \partial H_{i}(x)$, there exist scalars $\beta$ and $\gamma$ such that $v=\beta F_{i}^{\prime}(x)+\gamma G_{i}^{\prime}(x)$, where $\beta$ and $\gamma$ satisfy

(i) $\beta \gamma>0$ if $H_{i}(x) \neq 0$;

(ii) $\beta \neq 0 . \gamma=0$ if $H_{i}(x)=0$ and $F_{i}(x)=0, G_{i}(x)>0$,

(iii) $\beta=0, \gamma \neq 0$ if $H_{i}(x)=0$ and $F_{i}(x)>0, G_{i}(x)=0$,

(iv) $\beta \gamma \geq 0$ and $\beta+\gamma \neq 0$ if $H_{i}(x)=0, F_{i}(x)=G_{i}(x)=0$.

Now, we give the following method for (19).

Method 3.

Step 0: Choose $x^{0} \in \Omega$ and $\Gamma>0$. Let $k:=0$.

Step 1: If $x^{k} \in X$,then stop. 
Step 2: Solve the following linear programming problem to get $x^{k+1}$

$$
\min _{x, \gamma} \gamma
$$

$$
\begin{gathered}
\left\|H\left(x^{k}\right)+G\left(x^{k}\right)\left(x-x^{k}\right)\right\| \leq \gamma\left\|H\left(x^{k}\right)\right\|, \\
\left\|x-x^{k}\right\| \leq \gamma\left\|H\left(x^{k}\right)\right\|, \\
, \gamma \geq 0,
\end{gathered}
$$

where $G\left(x^{k}\right) \in \partial H\left(x^{k}\right)$. that is $x^{k+1} \in \mathrm{P}\left(x^{k}, \Gamma\right)$.

Step 3: Set $k:=k+1$. go to Step 1 .

Similar to the Section of 2.1, we give the following convergence theorem.

Theorem2. Let Assumptions 1-3 be satisfied for some $\delta>0$ and $\Gamma \geq \bar{\Gamma}$ be given. Then, if $x^{0} \in \beta\left(x^{*}, r_{\Gamma}\right) \cap \Omega$ for $r_{\Gamma} \in(0, \delta]$, then we have

(i) Method 3 is well-defined (that is, $\mathrm{P}\left(x^{k}, \Gamma\right) \neq \Phi$ in step 2 for all $k$ ).

(ii) For any $x^{0}, \ldots, x^{k}$ are generated by Method 3, then $x^{k+1} \in \beta\left(x^{*}, \delta\right) \cap \Omega$.

(iii) Any sequence $\left\{x^{k}\right\}$ generated by Method 3 either stops with a solution or converges locally quadratically to some solution of (21).

\section{DISCUSSION}

In this paper, we have give some applications of the Method 1, there are so many models can be solved by Method 1. We will give further study of this method in the future.

\section{ACKNOWLEDGEMENTS}

This work is supported by National Science Foundation of China (11101231).

Corresponding author. Shou-qiang Du, Email: qddsq1@163.com

\section{REFERENCES}

[1] F. Facchinei, A. Fischer, M. Herrich, A family Newton methods for nonsmooth constrained systems with nonisolated solutions[J], Mathematical Methods Operations Research, ,77:433-443, 2013.

[2] Y. Gao, New methods for solving two classes of nonsmooth equations [J], Applications of mathematics, 3:215-229,2007.

[3] F. Facchinei, A. Fischer, M. Herrich, An LP-Newton method: nonsmooth equations, KKT systems, and nonisolated solutions[J], Mathematical Programming, 146:1-36, 2014.

[4] D. F. Sun, L. Q. Qi, On NCP-Functions[J], Computational Optimization and Applications, 13:201-220, 1999.

[5] R. Behling, A. Fischer, A unified local convergence analysis of inexact constrained Levenberg-Marquardt methods[J], Optimization Letters, 6:927-940, 2012.

[6] A. Fischer, Solution of monotone complementarity problems with locally Lipschitzian functions[J], Mathematical Programming, 76:513532, 1997.
[7] D. F. Sun, J. Y. Han, Newton and quasi-Newton methods for a class of nonsmooth equations and related problems[J], SIAM Journal on Optimization, 7:463-480, 1997. 